\title{
Video Article \\ Electrophysiological Characterization of GFP-Expressing Cell Populations in the Intact Retina
}

\author{
Mark Pottek ${ }^{1}$, Gabriel C. Knop ${ }^{1}$, Reto Weiler ${ }^{1}$, Karin Dedek ${ }^{1}$ \\ ${ }^{1}$ Department of Neurobiology, University of Oldenburg \\ Correspondence to: Karin Dedek at karin.dedek@uni-oldenburg.de \\ URL: https://www.jove.com/video/3457 \\ DOI: doi:10.3791/3457
}

Keywords: Neuroscience, Issue 57, retina, transgenic mouse line, two-photon microscopy, patch-clamp recording, amacrine cells

Date Published: $11 / 14 / 2011$

Citation: Pottek, M., Knop, G.C., Weiler, R., Dedek, K. Electrophysiological Characterization of GFP-Expressing Cell Populations in the Intact Retina. J. Vis. Exp. (57), e3457, doi:10.3791/3457 (2011).

\section{Abstract}

Studying the physiological properties and synaptic connections of specific neurons in the intact tissue is a challenge for those cells that lack conspicuous morphological features or show a low population density. This applies particularly to retinal amacrine cells, an exceptionally multiform class of interneurons that comprise roughly 30 subtypes in mammals ${ }^{1}$. Though being a crucial part of the visual processing by shaping the retinal output ${ }^{2}$, most of these subtypes have not been studied up to now in a functional context because encountering these cells with a recording electrode is a rare event.

Recently, a multitude of transgenic mouse lines is available that express fluorescent markers like green fluorescent protein (GFP) under the control of promoters for membrane receptors or enzymes that are specific to only a subset of neurons in a given tissue ${ }^{3,4}$. These prelabeled cells are therefore accessible to directed microelectrode targeting under microscopic control, permitting the systematic study of their physiological properties in situ. However, excitation of fluorescent markers is accompanied by the risk of phototoxicity for the living tissue. In the retina, this approach is additionally hampered by the problem that excitation light causes appropriate stimulation of the photoreceptors, thus inflicting photopigment bleaching and transferring the retinal circuits into a light-adapted condition. These drawbacks are overcome by using infrared excitation delivered by a mode-locked laser in short pulses of the femtosecond range. Two-photon excitation provides energy sufficient for fluorophore excitation and at the same time restricts the excitation to a small tissue volume minimizing the hazards of photodamage ${ }^{5}$. Also, it leaves the retina responsive to visual stimuli since infrared light $(>850 \mathrm{~nm})$ is only poorly absorbed by photopigments ${ }^{6}$.

In this article we demonstrate the use of a transgenic mouse retina to attain electrophysiological in situ recordings from GFP-expressing cells that are visually targeted by two-photon excitation. The retina is prepared and maintained in darkness and can be subjected to optical stimuli which are projected through the condenser of the microscope (Figure 1). Patch-clamp recording of light responses can be combined with dye filling to reveal the morphology and to check for gap junction-mediated dye coupling to neighboring cells, so that the target cell can by studied on different experimental levels.

\section{Video Link}

The video component of this article can be found at https://www.jove.com/video/3457/

\section{Protocol}

The following description assumes that the experimenter has a basic understanding of retinal structure, patch-clamp recording, and two-photon microscopy. For useful information on establishing and running a patch-clamp setup and two-photon imaging system see Refs [7-12].

\section{Animal and tissue preparation}

1. Keep mouse dark-adapted for at least $3 \mathrm{~h}$. In the meantime, prepare $1-2 \mathrm{I}$ of extracellular solution consisting of (in $\mathrm{mM}$ ) $125 \mathrm{NaCl}, 2.5 \mathrm{KCl}$, 1 $\mathrm{CaCl}_{2}, 1.6 \mathrm{MgCl}_{2}, 25 \mathrm{NaHCO}_{3}, 10 \mathrm{D}$-glucose and equilibrate it to $\mathrm{pH} 7.4$ by gassing with carbogen $\left(5 \% \mathrm{CO}_{2}\right.$ in $\left.\mathrm{O}_{2}\right)$ at room temperature.

2. Euthanize the mouse in an air-tight chamber by $\mathrm{CO}_{2}$ overdose followed by cervical dislocation. This procedure and the following steps should be performed in darkness. Use dim long-wavelength illumination (we block short wavelengths from a cold light source by a $690 \mathrm{~nm}-$ longpass filter) to support personal vision while keeping the animal's eyes dark-adapted (mice only have poor vision in the red part of the light spectrum). For work in complete darkness use infrared illumination (>800 nm) and wear night-vision goggles.

3. Enucleate eyes with a pair of curved iris scissors and transfer them to a dish of extracellular solution placed under a dissecting microscope.

4. Remove the cornea and ciliary body by opening the eye bulb along the ora serrata (the border between retina and ciliary body) with a pair of spring scissors (we first use a lancet to pierce a starting point for the cutting). Take out the lens and carefully separate the retina from the pigment epithelium. If the orientation of the retina is crucial to your study, indicate it like in Ref [12]. Cut the optical nerve between retina and pigment epithelium and remove the retina from the eyecup. Note the direction of the retinal surfaces: the inside of the curled-up retina is the ganglion cell side; the outside is the photoreceptor side. 
5. Remove the vitreous from the inner retinal surface by gently pulling it off with the aid of a wooden toothpick. For this purpose, use a small volume of extracellular solution that just covers the retina. The vitreous sticks to the toothpick and can be dragged centrifugally off the retina. Then, apply short incisions along the retinal perimeter to facilitate flattening of the tissue.

6. Transfer the retina into the recording chamber photoreceptor-side down, spread it out on the glass bottom (we use a fine brush) and immobilize it with a nylon-strung frame of stainless steel. Prepare the second retina in the same way and keep it dark-adapted in carboxygenated extracellular solution for later use.

\section{Recordings}

1. Install the recording chamber in darkness under an upright laser scanning microscope and superfuse the retinal preparation continuously (not less than $5 \mathrm{ml} / \mathrm{min}$ ) with carboxygenated extracellular solution heated to $35^{\circ} \mathrm{C}$. The microscope (also equipped with an infrared-sensitive CCD camera) is situated on a shock-absorbing air table inside a Faraday cage for electronic shielding. Cover the cage with a non-transparent curtain to keep the preparation in darkness. We also screen off light from computer monitors by red transparent film.

2. Tune the infrared laser to $850-870 \mathrm{~nm}$ or longer wavelengths, switch to mode-locked condition, and use two-photon excitation to visualize GFP-expressing cells. Attenuate the laser output using neutral density filters controlled by the laser scanning software down to a degree that is just sufficient for clear recognition of the fluorescent cell

3. For patch-clamp recordings in current-clamp mode use glass micropipettes (we use borosilicate glass tubing of $1.5 \mathrm{~mm}$ outer diameter and $0.225 \mathrm{~mm}$ wall thickness) filled with intracellular solution consisting of (in mM) $125 \mathrm{~K}$-gluconate, $10 \mathrm{KCl}, 0.5 \mathrm{EGTA}, 10 \mathrm{HEPES}$, titrated to $\mathrm{pH} 7.4$ with $\mathrm{KOH}$ (giving a pipette resistance of about $5 \mathrm{M} \Omega$ ). Note that other experimental conditions like voltage-clamp recording require a different solution. If dye injections are desired, add a fluorescent probe (we use $10 \mathrm{mM}$ Alexa Fluor 594) or a tracer molecule (3\% Neurobiotin). Insert the micropipette into the holder and make sure that the reference electrode (chlorinated silver wire) is in contact with the extracellular solution in the recording chamber.

4. Apply pressure to the micropipette and target a GFP-expressing cell (we use a 40-fold water immersion objective; NA 1.25). Amacrine cell bodies are situated in the ganglion cell layer (directly beneath the surface in the current orientation of the retina) as well as in the proximal part of the inner nuclear layer (about 55-75 $\mu \mathrm{m}$ deep within the preparation). Before micropipette contact with the targeted cell, the inner limiting membrane (a sheath made of glial endfeet) at the retinal surface has to be penetrated. Successful penetration is recognized by intracellular solution that is driven out from the micropipette tip and detaches the inner limiting membrane from the underlying retinal tissue as can be observed on an infrared transmission image captured by the IR-CCD camera.

5. Approach the desired cell by comparing the soma position from the two-photon image with the picture of the IR-CCD camera showing the position of the recording micropipette. Note that this step is not easily achieved and requires some practice, because the GFP-expressing cell has to be recognized in the infrared transmission image in order to direct the micropipette towards it. Correct targeting is achieved when the micropipette tip causes dimpling of the cell surface that can be seen in the two-photon image. Alternative to this procedure, use a fluorescent dye (e.g. Alexa Fluor 594, $100 \mu \mathrm{M}$ ) in the intracellular solution to reveal the micropipette position in the two-photon image. Two-photon infrared excitation allows for sufficient fluorescence of this dye to make the micropipette discernible. For this purpose, adjust the detection range in the laser scanning software to cover also red fluorescence.

6. Release pressure from the micropipette and obtain a whole-cell patch-clamp configuration in current-clamp mode. A usable recording should give a membrane potential of -50 to $-55 \mathrm{mV}$ and last for at least $20 \mathrm{~min}$.

7. Present visual stimuli created by a stimulation software (we use QDS by Thomas Euler, University of Tübingen, Germany) ${ }^{11}$ on a computer monitor. Adjust the spatial position of the stimulation to be centered on the recorded cell: Mark the cell position on the monitor showing the transmission image of the IR-CCD camera and center a spot-like stimulus onto it. Tune the stimulus intensity by inserting neutral density filters into the beam path. For calibration of monitor spectrum and intensity see Ref [14]. Choose a prolonged interstimulus interval (e.g. $15 \mathrm{~s}$ ) to avoid adaptation. Include a photodiode within the beam path to keep record of the stimulus timing.

8. Start the stimulation protocol and record the light responses (we use a sampling rate of $10 \mathrm{kHz}$ with filtering at $5 \mathrm{kHz}$ for non-spiking cells; for spike recording a higher sampling rate like $20 \mathrm{kHz}$ is recommended). Use the light stimulation software to trigger the recording software.

9. For dye filling let the fluorescent agent diffuse into the recorded cell for 30-45 min before carefully retracting the micropipette from the cell. Take care not to pull out the cell body. If the non-fluorescent tracer Neurobiotin was used, it needs visualization by binding to streptavidin conjugated to a fluorophore (we use streptavidin-Cy3, 1:400 over night at $4{ }^{\circ} \mathrm{C}$ after 20 min paraformaldehyde fixation of the retina; for dyecoupling studies streptavidin incubation can be prolonged to $3 \mathrm{~d}$ ). Alternatively, injections can also be performed with sharp electrodes $(>100$ $\mathrm{M} \Omega$ ) when the dye is driven iontophoretically into the impaled cell (rectangular pulses: $0.25-1 \mathrm{nA}, 100 \mathrm{~ms}$, interval $100 \mathrm{~ms}$, total time 3-6 min).

\section{Representative Results:}

The following results originate from a study on a mouse expressing green fluorescent protein (GFP) under the promoter for tyrosine hydroxylase ${ }^{13,14}$, the enzyme catalyzing the rate limiting step in catecholamine synthesis (TH::GFP mouse). Based on the brightness of the GFPsignal two distinct cell populations are distinguishable (Figure 2A). Cells expressing the higher GFP level possess cell bodies located in the inner nuclear layer (INL, Figure 2A) or displaced in the ganglion cell layer (GCL, Figure 2B) and stratify in the middle of the inner plexiform layer (IPL, Figure 2C). They were identified as type 2 cells ${ }^{14-16}$ and could systematically be studied with respect to morphology (Figure 3 ) and electrical activity (Figure 4) even though its population density only amounts to $250 \mathrm{cells} / \mathrm{mm}^{2}$. 


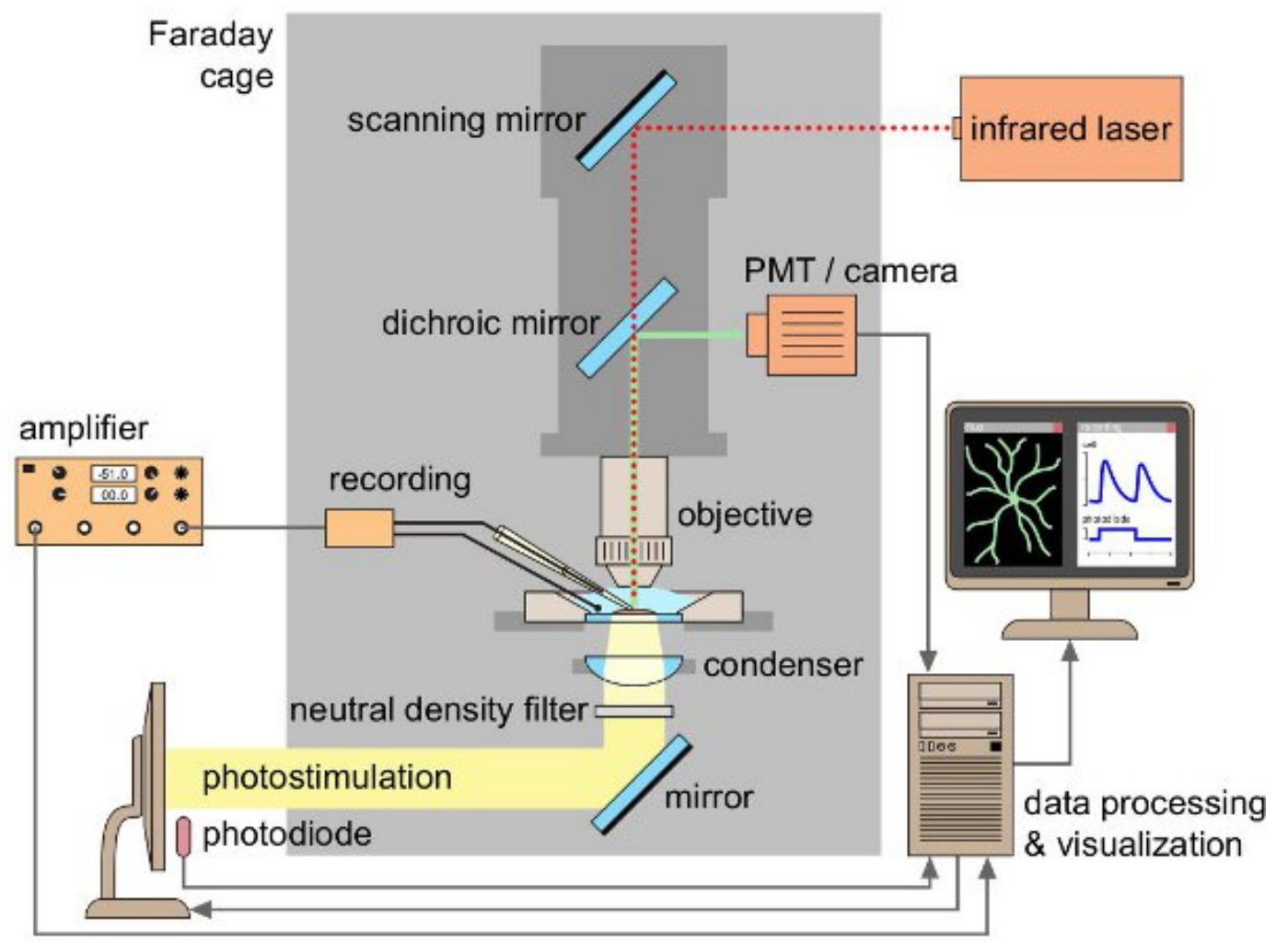

Figure 1. Schematic representation of the experimental setup. Two-photon excitation (red dotted light path) of fluorophore-expressing cells in the intact retina enables visual targeting by a micropipette (green emission light path). The retina is subjected to optical stimuli projected through the condenser of the microscope (yellow light path), and cellular light responses are recorded.
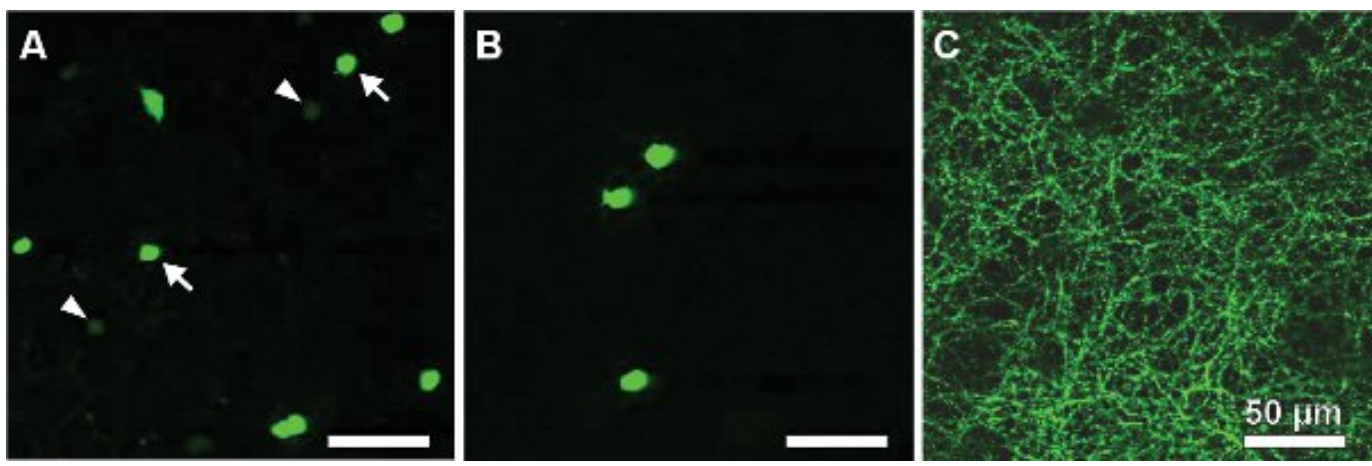

Figure 2. GFP-expressing cells in a retinal flatmount of a TH::GFP mouse. Two populations can be distinguished by the brightness of the GFPsignal: type 1 cells (DA cells) located in the INL showing weak fluorescence (A, see arrowheads) and intensely labeled type 2 cells with cell bodies either in the INL (A, see arrows) or displaced in the GCL (B) and a dendritic stratification in stratum S3 of the IPL (C). Scale bars, $50 \mu \mathrm{m}$. 


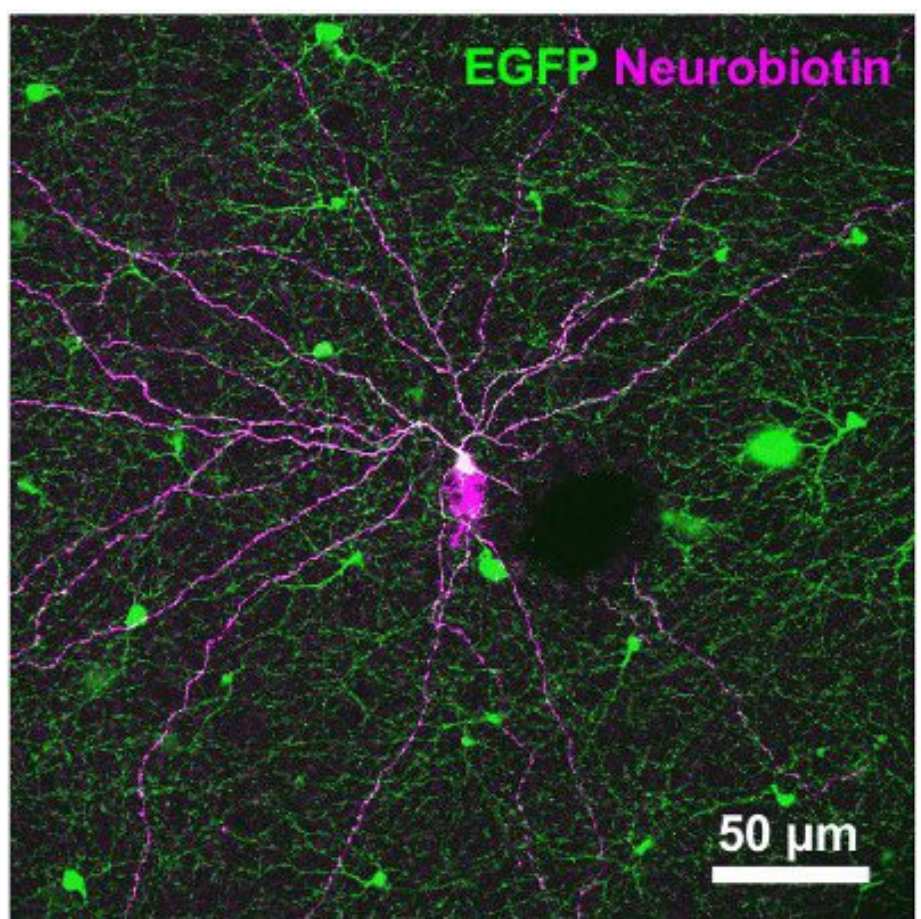

Figure 3.Morphology of a type 2 cell injected with the tracer Neurobiotin. The tracer was subsequently visualized by streptavidin-Cy3 binding (magenta). The micrograph, which is also depicting the GFP signal (green), is a projection of image stacks covering the GCL and IPL. Scale bar, $50 \mu \mathrm{m}$. 

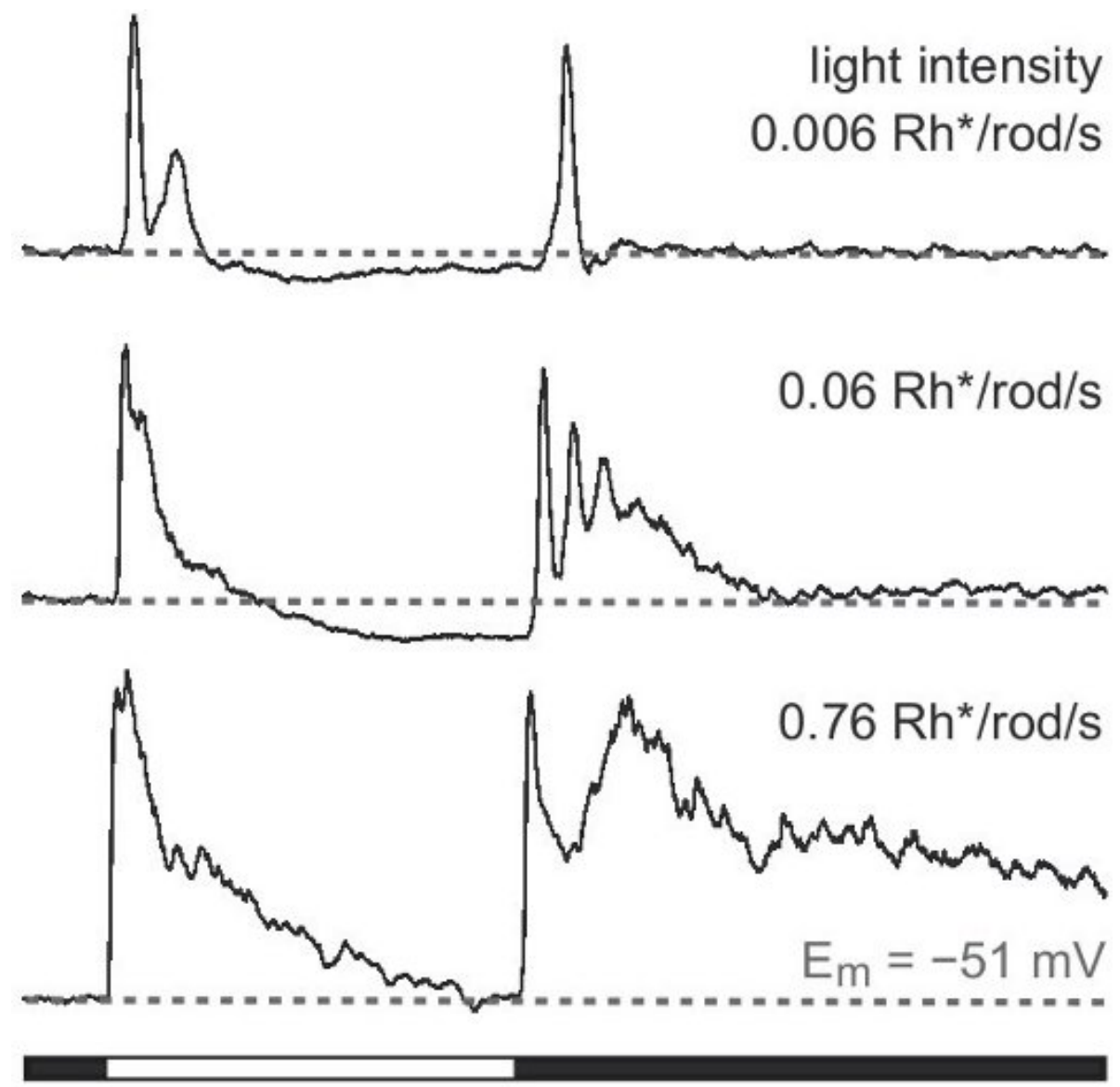

light stimulus (3 s)

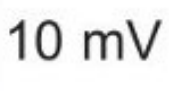

\section{$1 \mathrm{~s}$}

Figure 4. Light responses of a type 2 cell located in the GCL. Response pattern to white light full-field illumination of increasing intensity in the scotopic range. Stimulus intensity is given in photoisomerizations per rod per second ( $\mathrm{Rh} / / \mathrm{rod} / \mathrm{s})$. A prolonged stimulus of $3 \mathrm{~s}$ was used for better distinction of the response components at stimulus onset (ON response) and offset (OFF response).

\section{Discussion}

This method offers the possibility to study electrical properties of specific neurons in the intact retina under visual guidance without influencing the adaptational condition of the retina. It is particularly suited for the characterization of cells that are at present rather poorly studied due to low population density like most populations of amacrine cells. Two-photon excitation permits high-resolution and high-contrast imaging even from deeper parts of the tissue ${ }^{17}$, a prerequisite for accurate targeting and successful patch-clamping of cells particularly in the INL with its high density of cell bodies.

The isolated mouse retina is viable for 3-4 $\mathrm{h}$ under the experimental conditions. If the second retina is stored in complete darkness under continuous carbogen gassing, it retains the purple color of unbleached photopigment and can be used after finishing experiments with the first retina. In the beginning, targeting the selected cell with the micropipette in the retinal wholemount is a bit challenging and requires some practice, especially when working in darkness. Including a fluorescent dye in the micropipette can simplify the procedure, because cell and micropipette are visible under two-photon excitation at the same time. However, adding components to the intracellular solution may impede gigaseal formation or cause the recording quality to suffer. Once successfully achieved, a good recording can last for about $1 \mathrm{~h}$.

Whereas the infrared excitation light itself is only poorly absorbed by retinal photoreceptors, excited GFP-expressing cells emit light in the visible part of the spectrum. However, fluorophores are excited only in a small focal volume that is not likely to change the adaptational condition of the retina. All the more, excitation is only needed for targeting and can be switched off during recording of light responses.

The usefulness of this approach is already demonstrated by studies on specific populations of amacrine cells ${ }^{14,18}$ from which electrophysiological recordings otherwise would have been possible only by accident ${ }^{12}$. Finally, this powerful technique can further be extended by including a pharmacological approach ${ }^{14}, \mathrm{Ca}^{2+}$ imaging $^{19}$ or by using injected cells for immunocytochemical studies or electron microscopy. That way, the functional position of a given cell type in the retinal circuitry can be unraveled. 


\section{Disclosures}

Mice were handled and euthanized in accordance with institutional guidelines for animal welfare and the laws on animal experiments issued by the German government.

\section{Acknowledgements}

This work was supported by the Deutsche Forschungsgemeinschaft (WE849/16 1/2 to K.D. and R.W.). We are grateful to Thomas Euler (Töbingen, Germany) for the light stimulation software QDS.

\section{References}

1. Masland, R.H. The fundamental plan of the retina. Nat. Neurosci. 4, 877-886, (2001).

2. Baccus, S.A. Timing and computation in inner retinal circuitry. Annu. Rev. Physiol. 69, 271-290, (2007).

3. Haverkamp, S., Inta, D., Monyer, H., \& Wässle, H. Expression analysis of green fluorescent protein in retinal neurons of four transgenic mouse lines. Neuroscience. 160, 126-139, (2009).

4. Siegert, S., Gross-Scherf, B., Del Punta, K., Didkovsky, N., Heintz, N., \& Roska, B. Genetic address book for retinal cell types. Nat. Neurosci. 12, 1197-1204, (2009).

5. Svoboda, K. \& Yasuda, R. Principles of two-photon excitation microscopy and its applications to neuroscience. Neuron. 50, 823-839, (2006).

6. Euler, T., Detwiler, P.W., \& Denk, B. Directionally selective calcium signals in dendrites of starburst amacrine cells. Nature. 418, 845-852, (2002).

7. Sakmann, B. \& Neher, E. Single-Channel Recording. Plenum Press, (1995).

8. Jackson, M.B. Whole-cell voltage clamp recording. In: Current Protocols in Neuroscience., Gerfen, C., et al., eds. Wiley, (1997).

9. Majewska, A., Yiu, G., \& Yuste, R. A custom-made two-photon microscope and deconvolution system. Pfügers Arch. - Eur. J. Physiol. 441, 398-408, (2000).

10. Yuste, R. \& Konnerth, A. Imaging in Neuroscience and Development: A Laboratory Manual. (Cold Spring Harbor Laboratory, 2005).

11. Euler, T., Hausselt, S.E., Margolis, D.J., Breuninger, T., Castell, X., Detwiler, P.B., \& Denk, W. Eyecup scope - optical recordings of light stimulus-evoked fluorescence signals in the retina. Pflügers Arch. - Eur. J. Physiol. 457, 1393-1414, (2009).

12. Wei, W., Elstrott, J., \& Feller, M.B. Two-photon targeted recording of GFP-expressing neurons for light responses and live-cell imaging in the mouse retina. Nat. Protoc. 5, 1347-1352, (2010).

13. Matsushita, N., Okada, H., Yasoshima, Y., Takahashi, N., Kiuchi, K., \& Kobayashi, K. Dynamics of tyrosine hydroxylase promoter activity during midbrain dopaminergic neuron development. J. Neurochem. 82, 295-304, (2002).

14. Knop, G.C, Feigenspan, A., Weiler, R., \& Dedek, K. Inputs underlying the ON-OFF light responses of type 2 wide-field amacrine cells in THGFP mice. J. Neurosci. 31, 4780-4791, (2011).

15. Zhang, D.Q., Stone, J.F., Zhou, T., Ohta, H., \& McMahon, D.G. Characterization of genetically labeled catecholamine neurons in the mouse retina. Neuroreport. 15, 1761-1765, (2004).

16. Contini, M., Lin, B., Kobayashi, K., Okano, H., Masland, R.H., \& Raviola, E. Synaptic input to ON-biploar cells onto the dopaminergic neurons of the mouse retina. J. Comp. Neurol. 518, 2035-2050, (2010).

17. Helmchen, F. \& Denk, W. Deep tissue two-photon microscopy. Nat. Methods. 2, 932-940, (2005).

18. Dedek, K., Breuninger, T., de Sevilla Müller, L.P., Maxeiner, S., Schultz, K., Janssen-Bienhold, U., Willecke, K., Euler, T., \& Weiler, R. A novel type of interplexiform amacrine cell in the mouse retina. Eur. J. Neurosci. 30, 217-228, (2009).

19. Denk, W. \& Detwiler, P.B. Optical recording of light-evoked calcium signals in the functionally intact retina. Proc. Natl. Acad. Sci. U.S.A. 96, 7035-7040, (1999). 